\title{
The Rest-Frame Golenetskii Correlation via a Hierarchical Bayesian Analysis
}

\author{
J. Michael* \\ Max-Planck-Institut fÃijr extraterrestrische Physik \\ E-mail: jburgess ampe.mpg.de
}

\begin{abstract}
Gamma-ray bursts (GRBs) are characterised by a strong correlation between the instantaneous luminosity and the spectral peak energy within a burst. This correlation, which is known as the hardness-intensity correlation or the Golenetskii correlation, not only holds important clues to the physics of GRBs but is thought to have the potential to determine redshifts of bursts. In this paper, I use a hierarchical Bayesian model to study the universality of the rest-frame Golenetskii correlation and in particular, I assess its use as a redshift estimator for GRBs. I find that using a power-law prescription of the correlation, the power-law indices cluster near a common value, but have a broader variance than previously reported $(\sim 1-2)$. Furthermore, I find evidence that there is spread in intrinsic rest-frame correlation normalizations for the GRBs in our sample ( $\sim$ $10^{51}-10^{53} \mathrm{erg} \mathrm{s}^{1}$ ). This points towards variable physical settings of the emission (magnetic field strength, number of emitting electrons, photospheric radius, viewing angle, etc.). Subsequently, these results eliminate the Golenetskii correlation as a useful tool for redshift determination and hence a cosmological probe.
\end{abstract}

7th Fermi Symposium 2017

15-20 October 2017

Garmisch-Partenkirchen, Germany

${ }^{*}$ Speaker. 